\title{
Encefalopatia de Wernicke em criança com doença de Crohn
}

\author{
Wernicke encephalopathy in a child with Crohn disease
}

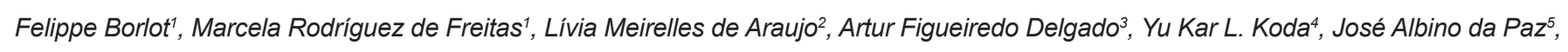
Maria Joaquina Marques-Dias ${ }^{6}$

\section{RESUMO}

Objetivo: Descrever um caso de encefalopatia de Wernicke associada à doença de Crohn na infância.

Descrição do caso: Menino de cinco anos, com diagnóstico de doença de Crohn por colonoscopia com biópsia há um ano; desde então, fez uso de diversos medicamentos sem resultados terapêuticos. Evoluiu com pancreatite há três meses, quando foi iniciada nutrição parenteral. Apresentou subitamente sonolência, episódios de frases desconexas e alteração da movimentação ocular. $\mathrm{O}$ exame neurológico evidenciou nistagmo vertical para cima em todas as posições e nistagmo horizontal no desvio conjugado do olhar. A ressonância magnética do encéfalo mostrou alterações compatíveis com a encefalopatia de Wernicke, sendo instituído o tratamento com tiamina parenteral imediatamente, com reversão completa dos sintomas por volta do $30^{\circ}$ dia de tratamento.

Comentários: Embora seja uma entidade rara, deve-se suspeitar de encefalopatia de Wernicke frente à presença de fatores de risco, uma vez que o tratamento imediato evita as sequelas neurológicas.

Palavras-chave: encefalopatia de Wernicke; doença de Crohn; criança; tiamina; nutrição parenteral.

\section{ABSTRACT}

Objective: To report a case of Wernicke encephalopathy associated with Crohn disease in childhood.

Case description: A five year-old boy with Crohn disease, diagnosed by colonoscopy and biopsy one year ago; he has been treated with many different medications without results. During the past year, the patient was diagnosed with pancreatites and has received parenteral nutrition since then. After three months, the child suddenly presented drowsiness, meaningless speech and ocular movement disturbance. Neurologic examination disclosed upbeat nystagmus in all positions and horizontal nystagmus during conjugate deviation of the eyes. Magnetic resonance showed abnormalities consistent with Wernicke encephalopathy. Parenteral thiamine has been administered soon after diagnosis and by the $30^{\text {th }}$ day of treatment, recovery of symptoms was complete.

Comments: Despite being a rare entity, neurological symptoms associated to the presence of risk factors such as prolonged parenteral nutrition should suggest Wernicke encephalopathy. Immediate treatment is important to avoid neurological disabilities.

Key-words: Wernicke encephalopathy; Crohn disease; child; thiamine; parenteral nutrition.
Instituição: Instituto da Criança do Hospital das Clínicas da Faculdade de Medicina da Universidade de São Paulo (ICr/HC-FMUSP), São Paulo, SP, Brasil ${ }^{1}$ Médico residente da Disciplina de Neurologia Infantil do HC-FMUSP, São Paulo, SP, Brasil

${ }^{2}$ Médica estagiária da Disciplina de Neurologia Infantil do HC-FMUSP, São Paulo, SP, Brasil

${ }^{3}$ Coordenador da Unidade de Terapia Intensiva do ICr/HC-FMUSP, São Paulo, SP, Brasil

${ }^{4}$ Coordenadora do Serviço de Gastroenterologia do ICr/HC-FMUSP, São Paulo, SP, Brasil

${ }^{5}$ Médico assistente da Disciplina de Neurologia Infantil do ICr/HC-FMUSP, São Paulo, SP, Brasil
${ }^{6}$ Livre-docente pela FMUSP; Professora-associada do Departamento de Neurologia e responsável pela Unidade de Neuropediatria do $\mathrm{ICr} / \mathrm{HC}$ FMUSP, São Paulo, SP, Brasil

Endereço para correspondência: Felippe Borlot

Av. Onze de Junho, 737/91 - Vila Clementino

CEP 04041-052 - São Paulo/SP

E-mail: felippe.borlot@icr.usp.br

Recebido em: 4/9/08

Aprovado em: 9/3/09 


\section{Introdução}

A encefalopatia de Wernicke (EW) é uma manifestação aguda atribuída à deficiência de vitamina B1 (tiamina), caracterizada pela tríade composta por oftalmoplegia, ataxia e alteração do estado mental. Dentre as alterações iniciais da movimentação ocular, destaca-se o nistagmo horizontal e/ou vertical, fraqueza ou paralisia dos músculos retos laterais e o olhar conjugado. A oftalmoplegia ocorre nos estágios mais avançados. Os distúrbios do estado mental podem variar desde distúrbios de atenção até déficit de memória, podendo evoluir mais tardiamente para o coma ${ }^{(1,2)}$.

A EW é mais frequente em adultos, particularmente nos usuários crônicos de álcool; na faixa etária pediátrica, destacam-se os pacientes com desnutrição crônica, neoplasias malignas, em uso prolongado de nutrição parenteral e, mais recentemente, a presença de distúrbios alimentares como a anorexia nervosa ${ }^{(3,4)}$. Na infância, existem dificuldades no diagnóstico clínico por se tratar de uma doença rara e porque a apresentação clínica nem sempre se faz com a presença da tríade clássica completa que, em crianças, ocorre em apenas $16 \%$ dos pacientes ${ }^{(5)}$. Não há relatos prévios de pacientes pediátricos portadores de doença de Crohn que apresentaram EW.

A ressonância magnética de encéfalo se tornou o método diagnóstico de escolha para a confirmação dos casos suspeitos devido às características peculiares lesionais e topográficas ${ }^{(1)}$. O tratamento com tiamina parenteral tem indicação absoluta e deve ser iniciada de forma rápida para fazer a profilaxia de sequelas neurológicas e impedir a evolução para a psicose de Korsakoff ${ }^{(6)}$.

Nesse contexto, o objetivo deste relato é descrever um caso de EW associada à doença de Crohn na infância, devido à sua raridade e à importância do diagnóstico e tratamento precoces, com redução da morbidade e mortalidade.

\section{Descrição do caso}

Menino de cinco anos e seis meses com antecedente de diarreia desde o nascimento, associada à dor abdominal e ao baixo ganho ponderal. Aos quatro anos, apresentou sangramento retal e, nessa ocasião, por meio de colonoscopia com biópsia, recebeu o diagnóstico de doença de Crohn, afetando do estômago ao reto. Fez uso de diversos esquemas medicamentosos, incluindo mesalazina $(60 \mathrm{mg} / \mathrm{kg} / \mathrm{dia})$, hidrocortisona $(10 \mathrm{mg} / \mathrm{kg} / \mathrm{dia})$ e azatioprina $(1,3 \mathrm{mg} / \mathrm{kg} / \mathrm{dia})$. Aos cinco anos e três meses, recebeu pulsoterapia com metilprednisolona sem resultados terapêuti- cos e, a seguir, por apresentar piora da diarreia e vômitos, foi internado e recebeu infusão de anticorpo monoclonal antifator necrose tumoral- $\alpha$. Pelos sintomas apresentados na ocasião, foi diagnosticada pancreatite e iniciada nutrição parenteral. $\mathrm{O}$ paciente foi transferido para o Instituto da Criança do Hospital das Clínicas da Faculdade de Medicina da Universidade de São Paulo (ICr/HC-FMUSP), onde recebeu a segunda dose de anticorpo monoclonal antifator necrose tumoral- $\alpha$; entretanto a criança evoluiu com sepse, distúrbios hidroeletrolíticos (acidose, hipofosfatemia e hipocalcemia) e endocardite, necessitando de terapia intensiva sem suporte ventilatório.

Aos cinco anos e seis meses, o paciente apresentou sonolência por 24 horas, alguns episódios de frases desconexas e alteração da movimentação ocular. Ao exame físico, apresentava-se em estado geral regular, descorado e emagrecido. O peso era de $13000 \mathrm{~g}$ (abaixo do percentil 3 para a idade) e a estatura de $106 \mathrm{~cm}$ (percentil 10 do comprimento para a idade). Observava-se edema bipalpebral discreto e sopro sistólico 2+/6+ em todo precórdio. O exame neurológico revelou criança chorosa, irritada, pouco colaborativa, emitindo apenas sons inarticulados e obedecendo apenas a comandos simples; não se interessava por objetos e apresentava diminuição global da movimentação espontânea, porém vencia a gravidade. Além disso, não foram evidenciados sinais motores de localização. Os reflexos miotáticos e o reflexo cutâneo-plantar estavam presentes e normais. Detectou-se nistagmo vertical para cima (upbeat) em todas as posições e nistagmo horizontal no desvio conjugado do olhar. O exame de fundo de olho foi normal. Realizou tomografia de crânio, que foi normal. A ressonância magnética do encéfalo mostrou hipersinal nos corpos mamilares, no putâmen e no tálamo mesial bilateral, nas sequências de T2 e no FLAIR (Figuras 1 e 2). Foi então levantada a hipótese diagnóstica de EW, com instituição imediata do tratamento com tiamina parenteral, na dose de 100mg/dia. Em 72 horas houve redução da amplitude do nistagmo e melhora do nível de consciência. A tiamina parenteral foi mantida durante duas semanas e a manutenção do tratamento realizada por via enteral na dose de $100 \mathrm{mg} / \mathrm{dia}(8 \mathrm{mg} / \mathrm{kg} / \mathrm{dia})$. Após 30 dias houve reversão completa do quadro neurológico. Na nova ressonância magnética realizada após três meses, houve desaparecimento das lesões (Figura 3).

\section{Discussão}

Na primeira descrição da EW na literatura, em 1881, Carl Wernicke relatou os casos de dois etilistas e de uma 


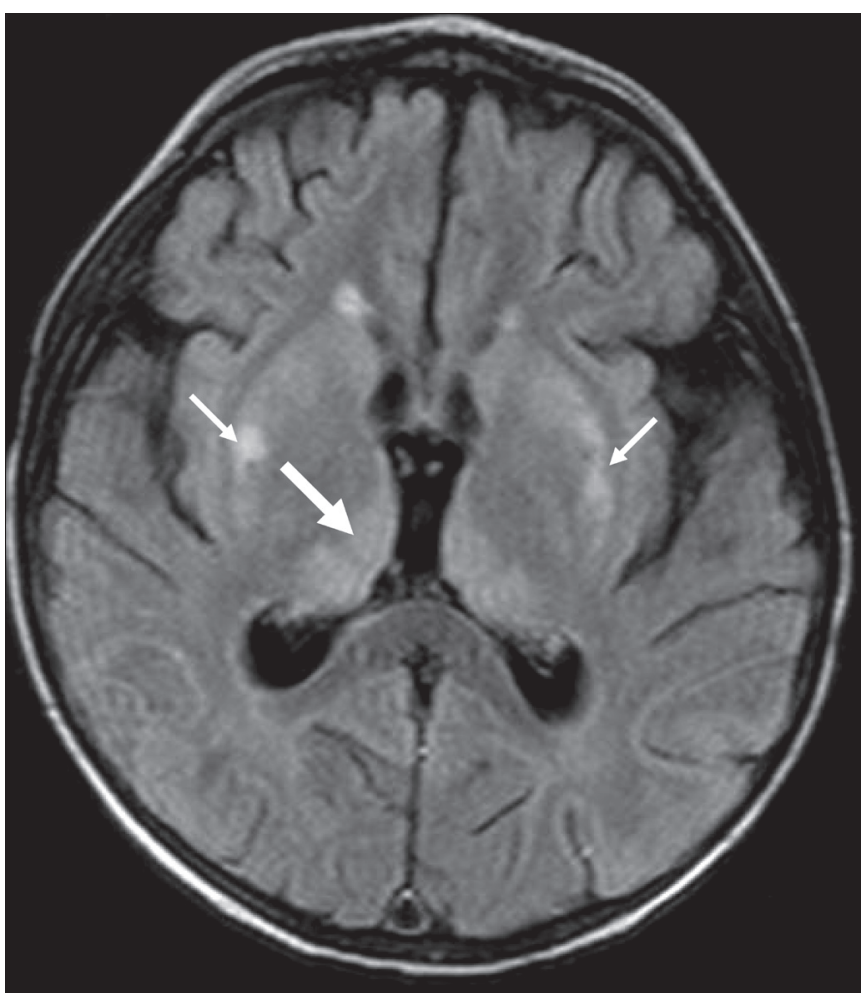

Figura 1 - Ressonância magnética em corte axial, sequência FLAIR: hipersinal intenso e simétrico nos tálamos, predominando na região mesial (seta grossa) e nos estriados (setas finas).

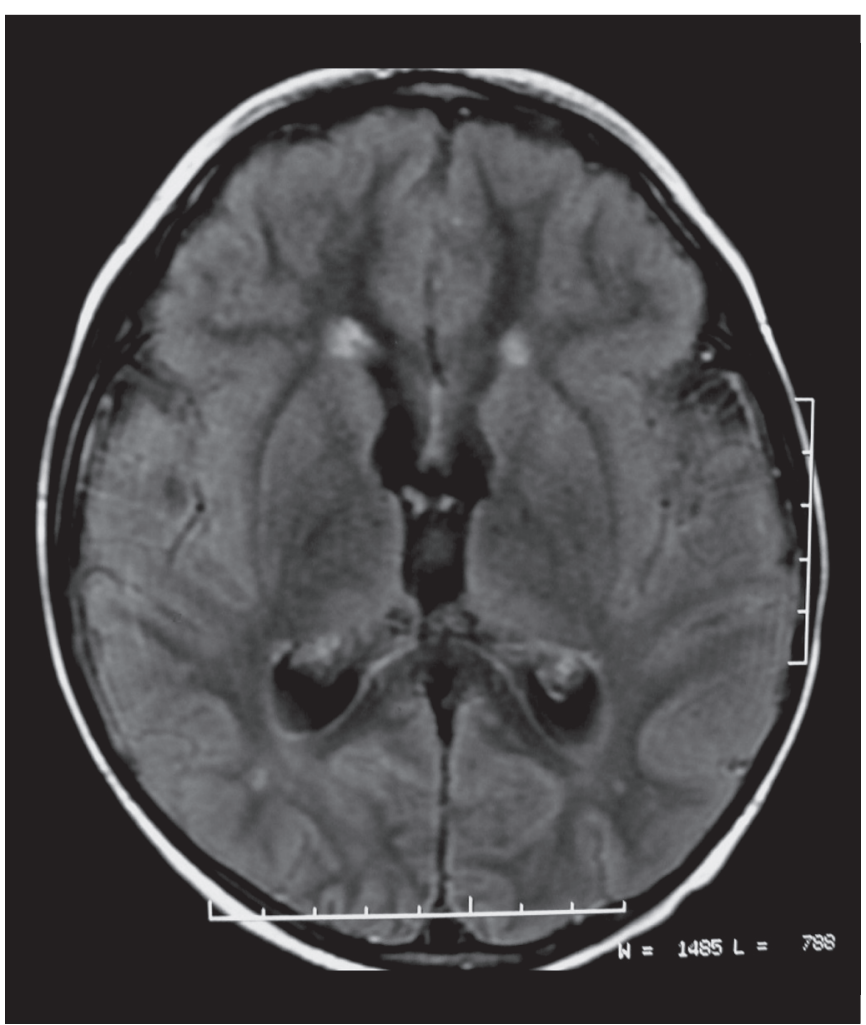

Figura 3 - Ressonância magnética em corte axial, sequência FLAIR: sem alterações.

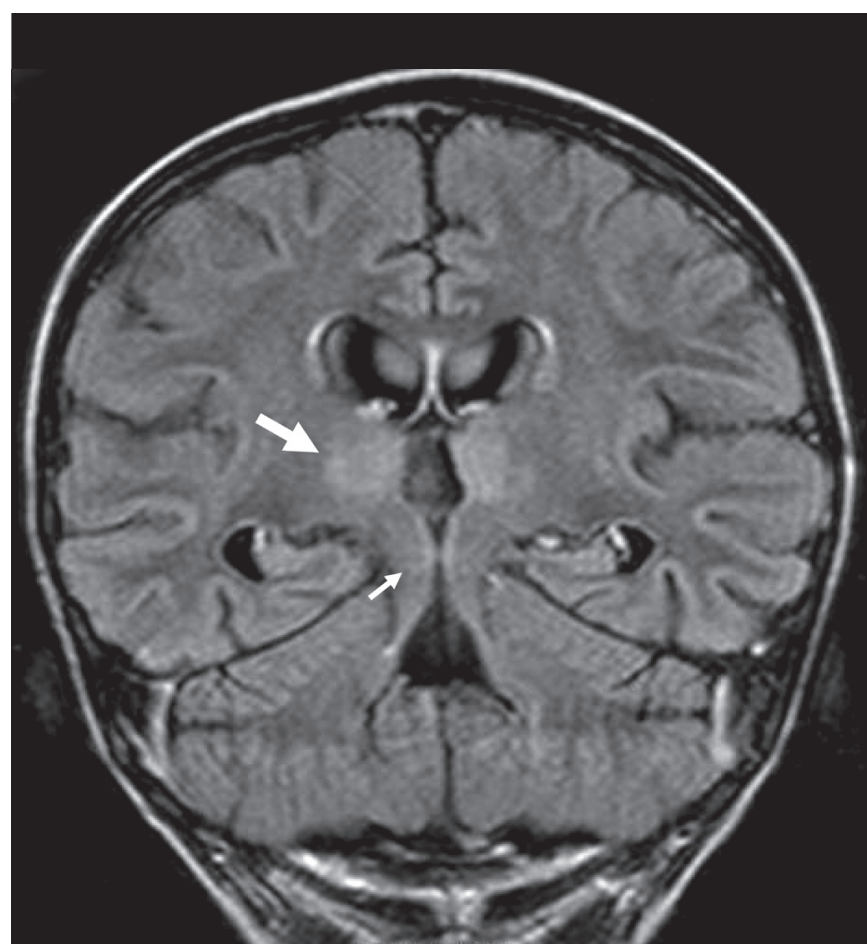

Figura 2 - Ressonância magnética em corte coronal, sequência FLAIR: hipersinal intenso e simétrico nos tálamos, predominando na região mesial (seta grossa) e hipersinal discreto na substancia cinzenta periaquedutal (seta fina).

paciente com vômitos persistentes, todos com alteração da movimentação ocular e do estado mental, além de ataxia; os pacientes faleceram no período de até duas semanas após o início dos sintomas neurológicos ${ }^{(3,7)}$. Posteriormente, entre 1930 e 1940, atribuiu-se à deficiência de vitamina B1 a causa da $\mathrm{EW}^{(8)}$. Se não tratados, os pacientes podem manifestar a psicose de Korsakoff, com confabulações e alterações na memória. Os sintomas iniciais podem não regredir e há evolução para coma ${ }^{(1,6,7)}$. O mecanismo patogênico ainda não está completamente elucidado, embora se saiba que a tiamina é uma enzima que participa do metabolismo intermediário dos carboidratos e tem importante papel na manutenção do gradiente osmótico através das membranas celulares. Sua deficiência cursa com edema intracelular no tecido cerebral e quebra da barreira hematoencefálica.

$\mathrm{Na}$ fase aguda, as alterações anatomopatológicas variam desde proliferação vascular - com proliferação da micróglia e dos astrócitos e hemorragia petequial - até necrose da substância cinzenta periquedutal, teto do quarto ventrículo, tálamo medial e corpos mamilares ${ }^{(1,3,6)}$. As anomalias detectadas pelos exames de neuroimagem se sobrepõem exatamente às alterações anatômicas, de forma bilateral e simétrica. A ressonância magnética destaca as lesões, com diminuição da 
intensidade do sinal na sequência $\mathrm{T} 1$, podendo haver realce após injeção do gadolínio; aparece aumento do sinal nas sequências T2 e FLAIR, sendo que na última há supressão do sinal do líquor nos ventrículos e nos espaços subaracnoides, facilitando assim a identificação de lesões periventriculares, do tronco e do tálamo ${ }^{(6,9)}$.

A administração de concentrações elevadas de glicose em pacientes com baixo estoque de tiamina pode precipitar a EW que, classicamente, é descrita em pacientes etilistas crônicos ou em desnutridos. Dentre outras causas citadas na literatura, destacam-se a hiperemese gravídica, o pós-operatatório de cirurgias gastrintestinais, o uso prolongado de nutrição parenteral com aporte inadequado de vitaminas, a doença de Crohn, a leucemia de células T, a síndrome da imunodeficiência adquirida, a hemodiálise e a anorexia nervosa ${ }^{(1,8)}$.

A dificuldade de diagnóstico da EW foi bem descrita em estudo retrospectivo de 131 casos, com análise de necropsia, o qual revelou que apenas $16 \%$ dos pacientes apresentaram a tríade clínica clássica, 37\% evidenciaram um único sinal, e $19 \%$ não mostraram quaisquer manifestações clínicas da EW. A alteração mental foi o sinal clínico mais comum em $74 \%$ dos pacientes ${ }^{(5)}$.

A revisão da literatura realizada por Vasconcelos $e t$ al em 1998 descreveu 31 pacientes com EW com idade abaixo de 20 anos: destes, 11 tinham antecedente de neoplasia maligna, 16 foram tratados com tiamina e oito permaneceram com sequelas. Os autores destacam o número de casos (42\%) com diagnóstico estabelecido apenas após a necrópsia ${ }^{(3)}$. No paciente aqui descrito, o diagnóstico diferencial mais próximo seria a

\section{Referências bibliográficas}

1. Hahn JS, Berquist W, Alcorn DM, Chamberlain L, Bass D. Wernicke encephalopathy and beriberi during total parenteral nutrition attributable to multivitamin infusion shortage. Pediatrics 1998;101:E10.

2. Zubaran C, Fernandes JG, Rodnight R. Wernicke-Korsakoff syndrome. Postgrad Med J 1997;73:27-31.

3. Vasconcelos MM, Silva KP, Vidal G, Silva AF, Domingues RC, Berditchevsky CR. Early diagnosis of pediatric Wernicke's encephalopathy. Pediatr Neurol 1999;20:289-94.

4. Wilson RK, Kuncl RW, Corse AM. Wernicke's encephalopathy: beyond alcoholism. Nat Clin Pract Neurol 2006;2:54-8.

5. Harper CG, Giles M, Finlay-Jones R. Clinical signs in the Wernicke-Korsakoff complex: a retrospective analysis of 131 cases diagnosed at necropsy. $\mathrm{J}$ Neurol Neurosurg Psychiatry 1986;49:341-5.

6. Harter SB, Nokes SR. Gadolinium-enhanced MR findings in a pediatric case of Wernicke encephalopathy. AJNR Am J Neuroradiol 1995;16:700-2. desmielinização após uso de anticorpo monoclonal antifator necrose tumoral- $\alpha$, utilizado na prática clínica atual em pacientes com artrite reumatóide e doença de Crohn. Especula-se que o anticorpo possa exacerbar a desmielinização, ativando as células T-específicas contra a mielina. Entretanto, os pacientes referidos na literatura foram expostos a doses repetidas dessa droga e apresentaram lesões, particularmente no sistema nervoso periférico, com evolução lenta para melhora após o uso de imunoglobulina; naqueles em que o sistema nervoso central foi afetado, as lesões eram inespecíficas e não eram similares às apresentadas pela criança em pauta ${ }^{(10,11)}$. O outro diagnóstico diferencial, pertinente ao caso principalmente pela topografia lesional no exame de ressonância magnética, seria o de mielinólise extrapontina. Entretanto, pelo fato de o paciente não ter alterações significativas dos eletrólitos séricos e apresentar evolução favorável, tal hipótese foi descartada.

$\mathrm{O}$ tratamento da EW consiste na infusão parenteral de vitamina $\mathrm{B} 1$ na dose de $100 \mathrm{mg}$ por no mínimo cinco dias, com manutenção em doses menores enquanto estiverem presentes os fatores de risco para a diminuição dos estoques de tiamina ${ }^{(4)}$. Após o uso da tiamina, os sintomas costumam reverter em algumas horas e a resolução completa demora até duas semanas. A mortalidade pode atingir $17 \%$ dos $\operatorname{casos}^{(1,7)}$. Assim, em crianças suscetíveis e diante de quaisquer dos sintomas da tríade clínica clássica da EW, essas devem ser avaliadas do ponto de vista neurológico e receber o tratamento adequado com tiamina, mesmo quando não há possibilidade de realizar a ressonância magnética, visando à profilaxia de sequelas neurológicas.

7. Victor A, Adams RD, Collins GH. The Wernicke-Korsakoff syndrome and related neurological disorders due alcoholism and malnutrition. $2^{\text {nd }}$ ed. Philadelphia: Davis; 1989.

8. Peters TE, Parvin M, Petersen C, Faircloth VC, Levine RL. A case report of Wernicke's encephalopathy in a pediatric patient with anorexia nervosa restricting type. J Adolesc Health 2007;40:376-83.

9. Zuccoli G, Gallucci M, Capellades J, Regnicolo L, Tumiati B, Giadás TC et al. Wernicke encephalopathy: MR findings at clinical presentation in twentysix alcoholic and non alcoholic patients. AJNR Am J Neuroradiol 2007;28: 1328-31.

10. Dubcenco E, Ottaway CA, Chen DL, Baker JP. Neurological symptoms suggestive of demyelination in Crohn's disease after infliximab therapy. Eur J Gastroenterol Hepatol 2006;18:565-6.

11. Jarand J, Zochodne DW, Martin LO, Voll C. Neurological complications of Infliximab. J Rheumatol 2006;33:1018-20. 